\title{
Особенности сорбционного извлечения 4-гидроксибензальдегида из водных растворов активированным углем Norit GAC 1240W
}

\author{
® 2021 Воронюк И.В. ${ }^{1}$, Елисеева Т.В. ${ }^{1}$, Свиридова Е.С. ${ }^{1}$, \\ Селеменев В.Ф. ${ }^{1}$, Сушкова А.А. ${ }^{1}$, Шолохова А.Ю. ${ }^{2}$ \\ ${ }^{1}$ Воронежский государственный университет, Воронеж \\ ${ }^{2}$ Институт физической химии и электрохимии им. А.Н. Фрумкина РАН, Москва, Россия
}

Поступила в редакцию 9.10.2020 г.

DOI: $10.17308 /$ sorpchrom.2021.21/3227

Активированные угли относятся к наиболее распространенным неорганическим сорбентам, активно используемым во многих областях промышленности с целью извлечения органических токсикантов. В настоящей работе проведено исследование особенностей сорбции представителя ряда замещенных бензальдегидов - пара-гидроксибензальдегида (ПГБА) - из водных растворов активированным углем Norit GAC1240W в статических условиях. Проведено сравнение равновесных характеристик сорбционного процесса с использованием товарного образца сорбента и предварительно растертого образца до порошкообразного состояния. Отмечен различный вид полученных зависимостей. Вследствие увеличения площади поверхности раздела на границе водный раствор ПГБА - сорбент и ее однородности наблюдается повышение сродства активированного угля к ароматическому альдегиду.

В работе проведена оценка изотерм сорбции с использованием формального подхода, основанного на подборе уравнений сорбции (Ленгмюра, Темкина, Фрейндлиха), максимально близко описывающих полученные зависимости. Отмечено, что наибольшие коэффициенты корреляции линейной зависимости для гранулированного сорбента отмечаются в результате представления кривой в координатах линеаризованного уравнения Фрейндлиха, для предварительно растертого - в координатах линеаризованного уравнения Ленгмюра. Представление зависимости емкости угля от равновесной концентрации гидроксибензальдегида в растворе в координатах уравнения мономолекулярной сорбции Ленгмюра позволило рассчитать некоторые равновесные характеристики исследуемого процесса (энергию сорбционного процесса, его энтальпию и энтропию). Отмечено, что поглощение пара-гидроксибензальдегида из раствора сопровождается экзотермическим эффектом. Кроме того, исследованы некоторые кинетические параметры сорбции - время достижения равновесия и константа скорости исследуемого процесса. Проведен анализ кинетической кривой сорбции ПГБА с позиции уравнений псевдо-первого, псевдо-второго порядков и Вебера-Мориса. Отмечено, что уравнение псевдо-второго порядка оптимально для описания полученной зависимости.

Таким образом, исследование сорбционного извлечения пара-гидроксибензальдегида из водных растворов с использованием активированного угля Norit GAC1240W позволило определить особенности равновесия и кинетики изучаемого процесса и оценить роль площади поверхности в случае молекулярной адсорбции данного соединения.

Ключевые слова: пара-гидроксибензальдегид, кинетика адсорбции, формальная кинетика, термодинамика адсорбции, активированный уголь.

\section{Введение}

Высокая сорбционная способность активированных углей обусловливает широкую область их применения во многих отраслях промышленности, в том числе для очистки сточных вод различных производств [1]. Авторами ранее исследована возможность использования анионообменных материалов для извлечения из водных растворов альдегидов алифа- 
тической природы, а также представителей гидроксибензальдегидов [2-4]. Механизм закрепления таких веществ многовариантен, однако основным путем закрепления сорбата в фазе сорбента является его взаимодействие с функциональной группой полимера. Этот факт обусловливает, с одной стороны, избирательность сорбции, с другой - невозможность участия в процессе поглощения всех функциональных групп ионообменника, что связано со стерическими трудностями при диффузии альдегида внутрь гранулы сорбента. Интерес вызывают неорганические сорбенты с привитыми функциональными группами. Такие сорбционные материалы имеют высокоразвитую поверхность, что увеличивает доступность групп, способных к взаимодействию с сорбатом. В отличие от полимерной гидрофобной матрицы ионообменных материалов неорганические сорбенты, в том числе угли, проявляют способность к молекулярной сорбции [5-7].

В настоящей работе проводилось установление особенностей кинетики сорбции пара-гидроксибензальдегида активированным углем Norit GAC $1240 \mathrm{~W}$ без привитых функциональных групп с целью оценки роли матрицы углеродного сорбента в процессе извлечения альдегидов ароматической природы.

\section{Экспериментальная часть}

В качестве объектов исследования выбраны водный раствор пара-гидроксибензальдегида (ПГБА) с концентрацией $6 \cdot 10^{-3}$ моль/дм ${ }^{3}$ и активированный уголь Norit GAC 1240 W с общей площадью поверхности $1100 \mathrm{~m}^{2} / \Gamma$ [8]. ПГБА в растворе определяли спектрофотометрически на приборе спектрофотометр «СФ2000» при длине волны 281 нм. Наличие в структуре сорбата гидроксигруппы обусловливает его слабокислотные свойства $(\mathrm{pK}=7.61$ [9]). Исследование особенностей сорбции ПГБА активированным углем проводили при $\mathrm{pH}<5.5$, что исключало возможность присутствия сорбата в анионной форме. Определение кинетических и равновесных характеристик сорбции осуществляли в статических условиях при постоянном перемешивании на орбитальном шейкере (220 об/мин), соотношение массы сорбента к массе раствора составляло 1:400.

\section{Обсуждение результатов}

В настоящей работе проведено исследование особенностей равновесия и кинетики сорбции в системе неорганический сорбент - водный раствор гидроксибензальдегида в статических условиях.

Равновесные характеристики сорбции ПГБА определяли методом переменных концентраций. На рис. 1 представлены

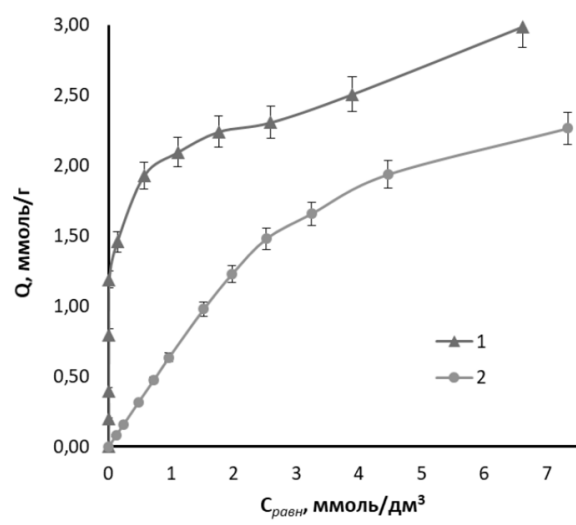

Рис. 1. Изотерма сорбции ПГБА активированным углем:

1 - предварительно растертый образец; 2 - товарный образец

Fig. 1. Sorption isotherm of PHBA by activated carbon:

1 - grounded sample; 2 - commercial sample 
Таблица 1. Коэффициенты корреляции $\left(\mathrm{R}^{2}\right)$ изотерм сорбции ПГБА активированным углем, представленных в линейных координатах уравнений сорбции

Table 1. Correlation coefficients $\left(\mathrm{R}^{2}\right)$ of sorption isotherms of PHBA by activated carbon, presented in linear coordinates of the sorption equations

\begin{tabular}{|c|c|c|c|}
\hline & $\begin{array}{c}\text { Уравнение типа } \\
\text { Ленгмюра }\end{array}$ & $\begin{array}{c}\text { Уравнение типа } \\
\text { Фрейндлиха }\end{array}$ & $\begin{array}{c}\text { Уравнение типа } \\
\text { Темкина }\end{array}$ \\
\hline $\begin{array}{c}\text { Предварительно } \\
\text { растертый образец угля }\end{array}$ & 0.995 & 0.819 & 0.956 \\
\hline Товарный образец угля & 0.931 & 0.978 & 0.925 \\
\hline
\end{tabular}

изотермы сорбции при использовании угля различной степени дисперсности: гранулированный и предварительно растертый образец сорбента. Отмечено, что в случае сорбции фенольного соединения на гранулированном образце на начальном участке полученной зависимости отмечается линейная зависимость емкости от количества сорбата в растворе. Очевидно, что отклонение от линейности на изотерме в области высоких концентраций сопряжено с насыщением поверхности угля пара-гидроксибенз-альдегидом. В то же время стоит отметить, что при переводе гранул неорганического сорбента в порошкообразное состояние площадь раздела на границе водный раствор ПГБА - активированный уголь увеличивается, что приводит к увеличению емкостных характеристик по сравнению с товарным образцом.

Вид изотерм сорбции гранулированного и порошкообразного Norit GAC1240W феноменологически относится к одному типу (I тип) по классификации IUPAC [10], но к разным типам (L2 и Н2, соответственно), согласно классификации Гильса [11]. Сорбция альдегида является мономолекулярной, при этом при использовании растертого сорбента повышается сродство к гидроксибензальдегиду, что в условиях одного механизма закрепления сорбата обусловлено увеличением площади поверхности угля и ее однородности.

Для оценки полученных равновесных характеристик сорбции использован формальный подход, основанный на анализе изотерм сорбции с позиции линеаризованных уравнений Ленгмюра Фрейндлиха (2) и Темкина (3) [12]:

$$
\begin{gathered}
\frac{C_{p a s H}}{Q}=\frac{1}{Q_{\max }} C_{p a s H}+\frac{1}{b \cdot Q_{\max }} \\
\ln Q=\ln \beta_{\phi}+\frac{1}{n} \ln C_{p a s H}, \\
F=\frac{1}{\alpha} \ln b+\frac{1}{\alpha} \ln C_{p a s H},
\end{gathered}
$$

где Q - величина сорбции при равновесной концентрации $\mathrm{C}_{\text {равн }}$, ммоль/г; $\mathrm{Q}_{\max }-$ величина предельной сорбции, соответствующая заполнению мономолекулярного слоя, $\mathrm{F}=\mathrm{Q} / \mathrm{Q}_{\max }$ - степень завершенности процесса, $\mathrm{b}$ - константа сорбционного равновесия; $\alpha, \beta_{\phi}$ и $\mathrm{n}-$ константы сорбции.

Представление полученных изотерм в координатах уравнений (1)-(3) позволило оценить применимость моделей сорбции по коэффициенту линеаризации полученных зависимостей (табл. 1).

Отмечено, что при исследовании сорбции на гранулированном сорбенте изотерма удовлетворительно описывается уравнением типа Фрейндлиха, а в случае растертого сорбента - уравнением типа Ленгмюра. Невысокие значения $\mathrm{R}^{2}$ не позволяют однозначно сделать заключение о механизме закрепления молекул пара-гидроксибензальдегида на поверхности угля, однако указывают на некоторые различия в поведении сорбента в разной степени дисперсности. Возможно, это связано с тем, что поверхность гранулированного угля более неоднородна, чем растертого.

В дальнейшей работе исследования проводились на образце растертого угля, позволяющего оценить максимально воз- 


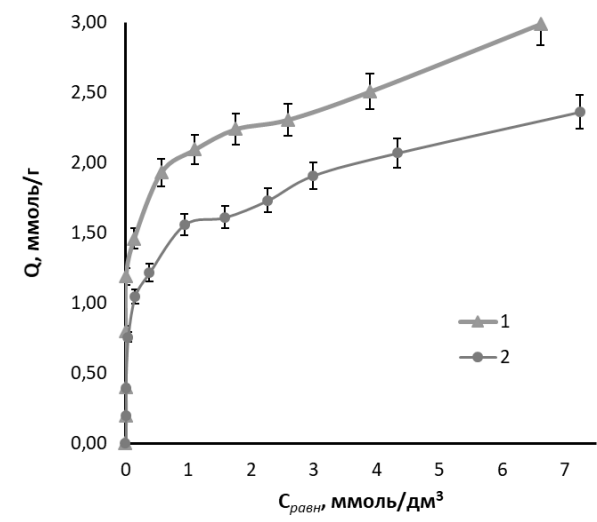

Рис. 2. Изотерма сорбции ПГБА активированным углем при различных темпеpaтурах: $1-298 \mathrm{~K}, 2-328 \mathrm{~K}$

Fig. 2. Sorption isotherm of PHBA by activated carbon at different temperatures:

$$
1-298 \mathrm{~K}, 2-328 \mathrm{~K}
$$

можную сорбционную способность к извлечению гидроксибензальдегида. Для оценки некоторых термодинамических характеристик сорбции получены изотермы сорбции ПГБА при различных температурах (рис. 2).

Отмечено, что с ростом температуры поглощение ПГБА на активированном угле уменьшается. Полученные зависимости позволили провести оценку термодинамических характеристик извлечения ароматического альдегида неорганическим сорбентом. Для этого в работе проводили линеаризацию изотерм сорбции в координатах уравнения типа Ленгмюра (1), которое позволило оценить величины констант сорбционного равновесия в зависимости от температуры системы.

По полученным данным проведен расчет кажущейся энергии сорбции ПГБА, энтальпии и энтропии сорбционного процесса (4)-(6).

$$
\begin{gathered}
\Delta G^{*}=-R T \ln b, \\
\Delta H=\frac{R T_{1} T_{2}}{T_{2}-T_{1}} \ln \left(\frac{b_{T_{2}}}{b_{T_{1}}}\right),
\end{gathered}
$$

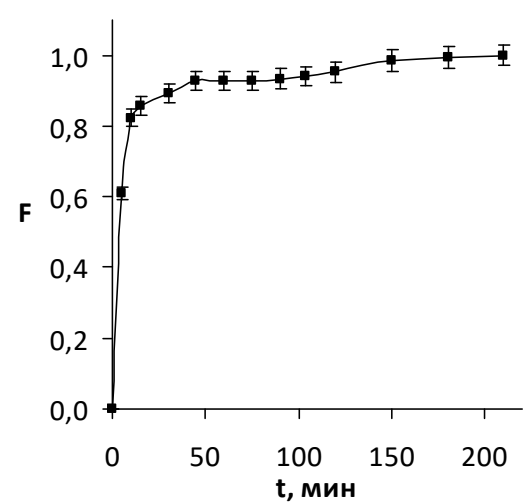

Рис. 3. Кинетическая кривая сорбции ПГБА активированным углем Norit GAC $1240 \mathrm{~W}\left(\mathrm{Co}=6 \cdot 10^{-3} \mathrm{M}\right)$

Fig. 3. Kinetic curve of the sorption of PHBA by activated carbon Norit GAC $1240 \mathrm{~W}\left(\mathrm{Co}=6 \cdot 10^{-3} \mathrm{M}\right)$

$$
\Delta S=\frac{\Delta H-\Delta G}{T},
$$

где $b_{T_{i}}$ - константа сорбционного равновесия альдегида при температуре системы $\mathrm{T}_{i}, \mathrm{~K}$. Полученные равновесные характеристики сорбции ПГБА активированным углем представлены в табл. 2 .

Энергия сорбции ПГБА свидетельствует о самопроизвольности процесса, при этом исследуемый процесс является экзотермическим.

Помимо равновесных характеристик сорбции в работе проведена оценка некоторых кинетических параметров исследуемого процесса. Отмечено, что время достижения равновесия в изучаемой системе составляет 150 минут (рис. 3).

Вид кинетической кривой сорбции пгидроксибензальдегида активированным углем указывает на высокую скорость поглощения на начальном участке (за 10 минут достигается степень завершенности процесса, равная 0.8), что связано с заполнением макропор угля, далее идет некоторое снижение скорости, что

Таблица 2. Термодинамические параметры сорбции ПГБА активированным углем Table 2. Thermodynamic parameters of sorption of PHBA by activated carbon

\begin{tabular}{|c|c|c|c|}
\hline Сорбент & $\Delta \mathrm{G}^{*}$, кДж/моль & $\Delta \mathrm{H}, \mathrm{\kappa Дж/моль}$ & $\mathrm{T} \Delta \mathrm{S}$, кДж/моль \\
\hline Norit GAC $1240 \mathrm{~W}$ & -24 & -17 & 7 \\
\hline
\end{tabular}


связано с заполнением микропор на поверхности угля.

Для описания полученных кинетических зависимостей сорбции ПГБА в работе использован формальный подход, основанный на представлении процесса сорбции как твердофазной реакции, протекающей на поверхности сорбент - раствор. Для описания таких взаимодействий могут использоваться модели кинетических уравнений псевдо-первого (7) [13], псевдо-второго порядков (8) [14], а также уравнения Вебера-Мориса (9) [15], устанавливающего влияние стадии внутренней диффузии на скорость поглощения вещества

$$
\begin{gathered}
\log \left(Q_{o}-Q_{t}\right)=\log \left(Q_{o}\right)-\frac{k_{p 1}}{2,303} t \\
\frac{t}{Q_{t}}=\frac{1}{k_{p 2} \cdot Q_{o}^{2}}+\frac{1}{Q_{o}} t
\end{gathered}
$$

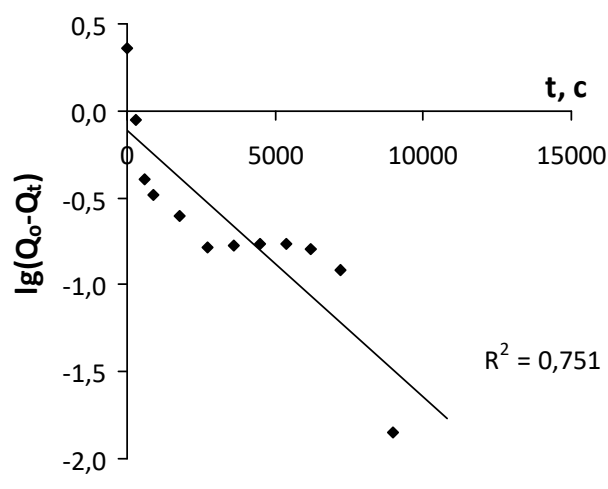

a

$$
Q_{t}=k_{i d} \cdot t^{0.5}+c
$$

где $Q_{o}, Q_{\mathrm{t}}$ - емкость сорбента в состоянии равновесия и в момент времени $\tau$, соответственно, ммоль/г; $\mathrm{kp}_{1}$ - константа скорости сорбции модели псевдо-первого порядка, $\mathrm{c}^{-1} ; \mathrm{k}_{\mathrm{p} 2}$ - константа скорости сорбции модели псевдо-второго порядка, $\left.\Gamma^{\cdot} \cdot(\text { ммоль })^{-1}\right)^{-1} ; k_{\text {id }}-$ константа скорости внутренней диффузии, ммоль/(г·c $\left.c^{0.5}\right) ; \mathrm{c}-$ параметр, характеризующий влияние пограничного слоя на скорость сорбции.

Для выбора уравнения химической кинетики, наиболее оптимально описывающего полученные кинетические кривые, проводили их линеаризацию в координатах этих уравнений (7)-(8). Полученные зависимости представлены на рис. 4.

Наибольший коэффициент корреляции наблюдается для кинетической кри-
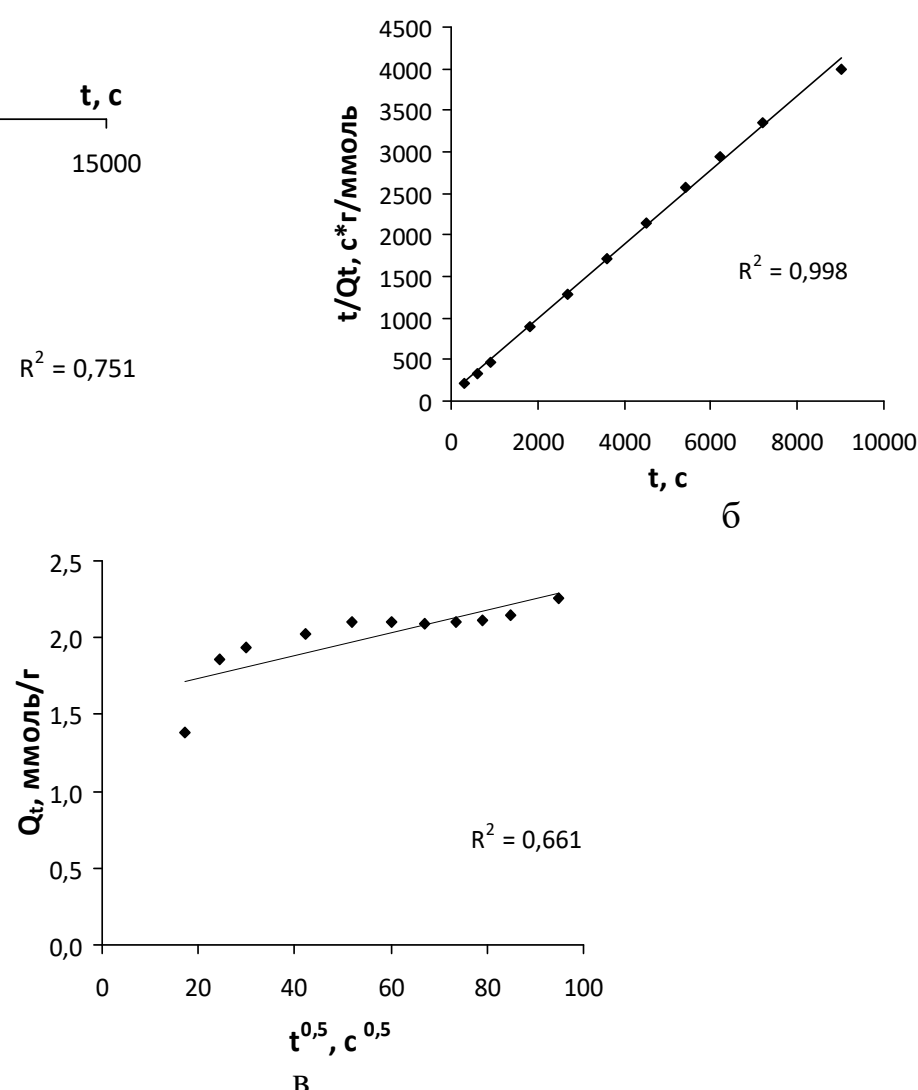

Рис. 4. Кинетические кривые сорбции ПГБА активированным углем в линейных координатах уравнений: а - псевдо-первого порядка, б - псевдо-второго порядка, в - модифицированной модели Вебера-Мориса

Fig. 4. Kinetic curves of PHBA sorption by activated carbon in linear coordinates of the equations of: $a$ - pseudo-first order, $b$ - pseudo-second order, $\mathrm{c}$ - modified Weber-Morris model 
вой, представленной в координатах уравнения псевдо-второго порядка. Решение данного уравнения графическим путем позволило рассчитать константы скорости сорбции ПГБА сорбентом неорганической природы $-3.7 \cdot 10^{-5} \Gamma \cdot(\text { ммоль } \cdot)^{-1}$.

Из литературных источников известно, что при сорбции большинства органических соединений неорганическими сорбентами наблюдается линейность в координатах уравнения именно псевдовторого порядка [16-18]. Данный факт свидетельствует о влиянии скорости взаимодействия между сорбентом и сорбатом на скорость всего сорбционного процесса. Полученные величины констант скорости поглощения ПГБА имеют один порядок с аналогичными величинами при сорбции фенола [17] и бензальдегида [16].

\section{Заключение}

Исследование равновесных и кинетических характеристик сорбции п-гидроксибензальдегида активированным углем Norit GAC1240W позволило установить ряд особенностей. Ввиду того, что поглощение на поверхности неорганического сорбента осуществляется преимущественно по механизму молекулярной ад-

сорбции, определяющим фактором сорбционной емкости является площадь границы раздела контактирующих фаз. Предварительное измельчение образца позволяет добиться более высокой адсорбционной способности к изучаемому фенольному соединению и увеличить скорость процесса. При этом степень дисперсности угля будет оказывать существенное влияние на коэффициенты распределения ПГБА в сорбенте. При оценке изотерм сорбции гидроксибензальдегида активированным углем при различной температуре отмечено, что исследуемый процесс экзотермичен, причем на энергию сорбции в большей мере оказывает влияние энтальпийная составляющая процесса.

Кинетическая кривая сорбции с позиции формального подхода может быть описана с использованием уравнения псевдо-второго порядка, при этом константа скорости исследуемого процесса составляет $3.7 \cdot 10^{-5} \Gamma \cdot(\text { ммоль } \cdot \text { с })^{-1}$.

Таким образом, активированный уголь Norit GAC1240W проявляет способность к извлечению пара-гидроксибензальдегида по механизму молекулярной адсорбции, показывая высокую скорость поглощения и значительную емкость по отношению к исследуемому сорбату.

Работа выполнена при поддержке Министерства науки и высшего образования РФ в рамках государственного задания ВУЗам в сфере научной деятельности на 2020-2022 годbl, проект № FZGU-2020-0044»

\section{Список литературы}

1. Мухин В.М., Клушин В.Н. Производство и применение углеродных адсорбентов. M. Российский химико-технологический университет им. Д.И. Менделеева. 2012. 305 c.

2. Воронюк И.В., Елисеева Т.В. // Сорбиионные и хроматографические прочессы. 2018. T. 18. № 2. C. 231-237

3. Решетникова О.В., Яцев А.М., Воронюк И.В., Елисеева Т.В. // Сорбиионные и хроматографические прочессы. 2015. Т. 15. № 4. С. 571-577.
4. Шолохова А.Ю., Елисеева Т.В., Буряк А.К. // Коллоидный журнал. 2019. Т. 81. № 5. C. $676-680$.

5. Котельникова Т.А., Кузнецов Б.В., Морева А.А., Муравьева Г.П. // Сорбиионные и хроматографические проиессы. 2012. Т. 12. №. 2. C. 295-303.

6. Koyuncu H. // Applied Clay Science. 2008. Vol. 38. No. 3-4, pp. 279-287.

7. Кохно Г.В., Хохлова Г.П. // Вестник Кузбасского государственного технического университета. 2007. № 6. С. 123-125. 
8. Norit digital library. (http://www.irimex.ru /files/catalog/rubs/files/887/3.pdf) (дата обращения: 28.09.2020).

9. P-Hydroxybenzaldehyde

(https://go. drugbank.com/drugs/DB03560) (дата обращения: 28.09.2020).

10. Handbook of heterogeneous catalysis. Wiley-VCH Verlag GmbH\& Co. KGaA, Weinheim, Germany. Second Completely Revised and Enlarged Edition, Vol. 1.2008, 3865 p.

11.Giles C.H., MacEwan C.H., Nakhwa S.N., Smith D. // J. Chem. Soc. 1960. pp. 3973-3993.

12.Адсорбция из растворов на поверхности твердых тел / Ред. Г. Парфит, К. Рочестер. М. Мир. 1986. 488 с.

13.Lagergren S. // K. Sven. Vetenskapsakad. Handl. 1898. Vol. 24. No 4. pp. 1-39.
14.Ho Y.S., McKay G. // Process Biochem. 1999. Vol. 34. pp. 451-465.

15.Weber J.G., Asce J.M., Morris J.C. // J. Sanit. Eng. Div. Am. Soc. Civ. Engrs. 1963. Vol. 89. pp. 31-59.

16.Rajoriya R.K., Prasad B., Indramani M., Kailas L. W. // Chem. Biochem. Eng. 2007. No 21. pp. 219-226.

17.Сивакова Л.Г. // Вестник Кузбасского государственного технического универсиmema. 2007. № 2. С. 94-96.

18.Романцова И.В., Бураков А.Е., Кучерова А.Е. // Известия Самарского научного иентра Российской академии наук. 2014. Т. 16. № 4. C. 611-614.

\title{
Features of sorption of 4-hydroxybenzaldehyde from aqueous solutions by Norit GAC 1240W activated carbon
}

\author{
(C) 2021 Voronyuk I.V. ${ }^{1}$, Eliseeva T.V. ${ }^{1}$, Sviridova E.S. ${ }^{1}$, Selemenev V.F. ${ }^{1}$, \\ Sushkova A.A. ${ }^{1}$, Sholokhova A.Yu. ${ }^{2}$ \\ ${ }^{1}$ Voronezh State University, Voronezh, Russian Federation \\ ${ }^{2}$ A.N.Frumkin Institute of Physical Chemistry and Electrochemistry of the Russian Academy of Sciences, \\ Moscow, Russian Federation
}

\begin{abstract}
Activated carbons are among the most widespread inorganic sorbents, actively used in many areas of industry to extract organic toxicants. In this study, we investigated the features of sorption of a representative of a number of substituted benzaldehydes, para-hydroxybenzaldehyde (PHBA), from aqueous solutions by Norit GAC1240W activated carbon under static conditions. A comparison of the equilibrium characteristics of the sorption process was carried out using a commercial sample of the sorbent and the sample that was ground to powder. A different form of the obtained dependences was noted. An increase in the affinity of activated carbon to aromatic aldehyde was observed due to an increase in the area of interface between the aqueous solution of PHBA and the sorbent, as well as the uniformity of the interface.

The study evaluates the sorption isotherms using a formal approach based on the selection of sorption equations (Langmuir, Temkin, Freundlich) describing the obtained dependences as closely as possible. It has been noted that the highest correlation coefficients of the linear dependence for the granular sorbent were noted as a result of the presentation of the curve in the coordinates of the linearized Freundlich equation, for the ground sorbent - in the coordinates of the linearized Langmuir equation. Representation of the dependence of the capacity of carbon on the equilibrium concentration of hydroxybenzaldehyde in the solution in the coordinates of the Langmuir monomolecular sorption equation allowed calculating some equilibrium characteristics of the studied process (the energy of the sorption process, its enthalpy and entropy). It was noted that the adsorption of para-hydroxybenzaldehyde from the solution is accompanied by an exothermic effect. In addition, some kinetic parameters of sorption were investigated - the time of achieving equilibrium and the rate constant of the studied process. The analysis of the kinetic curve of sorption of PHBA was carried out from the position of the pseudo-first, pseudo-second order equations, and Weber-Morris equation. It was noted that the pseudo-second order equation is optimal for describing the obtained dependence.

Thus, the study of the sorption extraction of para-hydroxybenzaldehyde from aqueous solutions using Norit GAC1240W activated carbon allowed determining the features of the equilibrium and kinetics of the studied process and evaluating the role of the surface area in the molecular adsorption of this compound.

Keywords: para-hydroxybenzaldehyde, adsorption kinetics, formal kinetics, adsorption thermodynamics, activated carbon
\end{abstract}




\section{References}

1. Mukhin V.M., Klushin V.N., Proizvodstvo i primenenie uglerodnyh adsorbentov, M., Rossiyskiy himiko-tekhnologicheskiy universitet im. D.I. Mendeleeva, 2012, 305 p.

2. Voronyuk I.V., Eliseeva T.V., Sorbtsionnye i khromatograficheskie protsessy, 2018, Vol. 18, No 2, pp. 231-237

3. Reshetnikova O.V., Yatsev A.M., Voronyuk I.V., Eliseeva T.V., Sorbtsionnye $i$ khromatograficheskie protsessy, 2015, Vol. 15, No 4, pp. 571-577.

4. Sholohova, A.Yu., Eliseeva T.V., Buryak A.K., Kolloidnyy zhurnal, 2019, Vol. 81, No 5, pp. 676-680.

5. Kotel'nikova T.A., Kuznetsov B.V., Moreva A.A., Murav'eva G.P., Sorbtsionnye i khromatograficheskie protsessy, 2012, Vol. 12, No 2, pp. 295-303.

6. Koyuncu H., Applied Clay Science, 2008, Vol. 38, No 3-4, pp. 279-287.

7. Kohno G.V., Khokhlova G.P., Vestnik Kuzbasskogo gosudarstvennogo tekhnicheskogo universiteta, 2007, No 6, pp. 123-125.

8. Norit digital library. (http://www.irimex.ru/files/cata$\log /$ rubs/files/887/3.pdf) (data obrashcheniya: 28.09.2020).

Воронюк Ираида Владимировна - к.Х.Н., доцент кафедры аналитической химии ФГБОУ $\mathrm{BO}$ «Воронежский государственный университет», Воронеж

Елисеева Татьяна Викторовна - к.Х.Н., зав. кафедрой аналитической химии ФГБОУ ВО «Воронежский государственный университет», Воронеж

Свиридова Елена Сергеевна - студентка магистратуры кафедры аналитической химии ФГБОУ ВО «Воронежский государственный университет», Воронеж

Селеменев Владимир Федорович - д.Х.н., проф. каф. аналитической химии, Воронежский государственный университет, Воронеж

Сушкова Анастасия Альбертовна - студентка магистратуры кафедры аналитической химии ФГБОУ ВО «Воронежский государственный университет», Воронеж

Шолохова Анастасия Юрьевна - м.н.с. лаборатории физико-химических основ хроматографии и хромато-масс-спектрометрии, Институт физической химии и электрохимии имени А.Н. Фрумкина РАН, Москва
9. p-Hydroxybenzaldehyde (https://go. drugbank.com/drugs/DB03560) (data obrashche-niya: 28.09.2020).

10. Handbook of heterogeneous catalysis, Wiley-VCH Verlag GmbH\& Co. KGaA, Weinheim, Germany. Second Completely Revised and Enlarged Edition, Vol. 1, 2008, 3865 p.

11. Giles C.H., MacEwan C.H., Nakhwa S.N., Smith D., J. Chem. Soc., 1960, pp. 3973-3993.

12. Adsorbtsiya iz rastvorov na poverhnosti tverdyh tel, Red. G. Parfit, K. Rochester, M., Mir, 1986, 488 p.

13. Lagergren S., Sven K., Vetenskapsakad. Handl, 1898, Vol. 24, No 4, pp. 1-39.

14. Ho Y.S., McKay G., Process Biochem., 1999, Vol. 34, pp. 451-465.

15. Weber J.G., Asce J.M., Morris J.C., J. Sanit. Eng. Div. Am. Soc. Civ. Engrs., 1963, Vol. 89, pp. 31-59.

16. Rajoriya R.K., Prasad B., Indramani M., Kailas L. W., Chem. Bio-chem. Eng., 2007, No 21, pp. 219-226.

17. Sivakova L.G., Vestnik Kuzbasskogo gosudarstvennogo tekhnicheskogo uni-versiteta, 2007, No 2, pp. 94-96.

18. Romantsova I.V., Burakov A.E., Kucherova A.E., Izvestiya Samarskogo nauchnogo tsentra Rossiyskoy akademii nauk, 2014, Vol. 16, No 4, pp. 611-614.

Voronyuk Iraida V. - assistant professor, Department of Analytical Chemistry, Voronezh State University, Voronezh, e-mail: i.voronyuk@yandex.ru

Eliseeva Tatiana V. - head of Department of Analytical Chemistry, Voronezh State University, Voronezh, e-mail:tatyanaeliseeva@yandex.ru

Sviridova Elena S. - Master student, Department of Analytical Chemistry, Voronezh State University

Selemenev Vladimir F. - DSci in chemistry, Voronezh State University, Voronezh, Russian Federation, e-mail: common@.chem.vsu.ru

Sushkova Anastasiya A. - Master student, Department of Analytical Chemistry, Voronezh State University, e-mail: sushkova.nastasya31@mail.ru

Sholokhova Anastasiya Yu. - junior researcher, laboratory of physical-chemical basics of chromatography and chromato-mass-spectrometry, Institute of Physical chemistry and electrochemistry, e-mail: shonastya@yandex.ru 